\title{
STAR FORMATION AT HIGH ANGULAR RESOLUTION
}

Edited by: MICHAEL BURTON, RAY JAYAWARDHANA and TYLER BOURKE
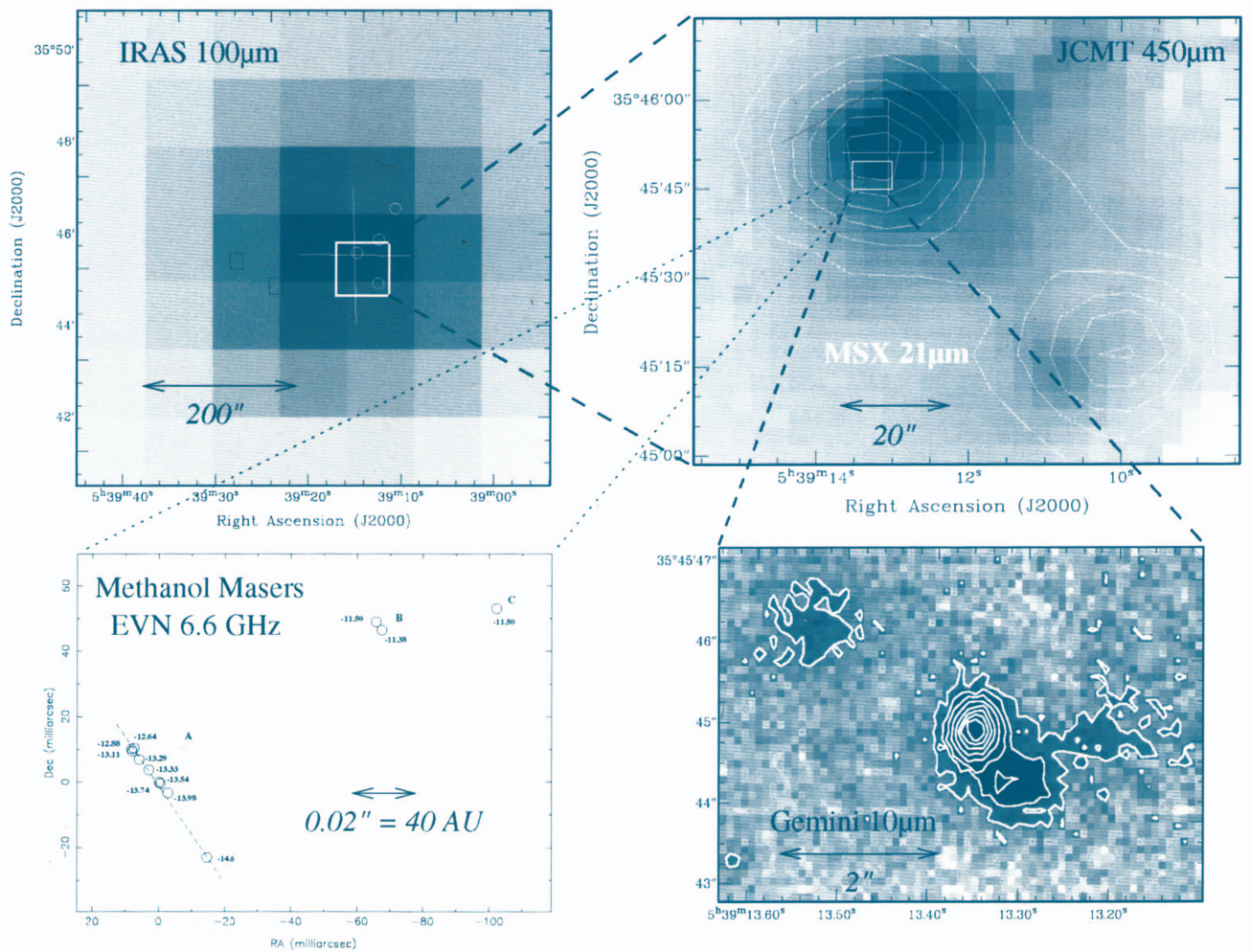

\section{$\Psi \mathbb{A} \mathbb{U}$}

INTERNATIONAL ASTRONOMICAL UNION

PUBLISHER:

ASTRONOMICAL SOCIETY OF THE PACIFIC 


\section{Cover Illustration:}

Revealing star formation through increasing angular resolution in the source G173.49+2.42 (S231-IR). Top-left is the IRAS $100 \mu \mathrm{m}$ image of this $5 \times 10^{4} \mathrm{~L}_{\text {。 }}$ embedded object $1,800 \mathrm{pc}$ away, harbouring a massive protostellar core. The position of a methanol maser is marked with a cross, and two UCHII regions with squares. Four sub-millimetre sources are associated with the IRAS source (circles), and two of these are illustrated in the higher angular resolution view to top-right. This shows the JCMT image at $450 \mu \mathrm{m}$ overlaid with contours of $21 \mu \mathrm{m}$ emission, as imaged with the $M S X$ satellite. The methanol maser is coincident with the brightest sub-mm and mid-IR core. Imaging this core with Gemini at $10 \mu \mathrm{m}$ at arcsecond resolution resolves it into at least four sources (bottom-right). Probing it at milli-arcsecond resolution with the European VLBI Network (EVN) shows three clusters of methanol masers (bottom-left), with a clear $2 \mathrm{~km} / \mathrm{s}$ velocity gradient over the $100 \mathrm{AU}$ extent of the eastern maser cluster. Scale bars show the relative angular resolution of each frame.

Data provided by: Vincent Minier, Steven Longmore and Michael Burton. 
ASTRONOMICAL SOCIETY OF THE PACIFIC

390 Ashton Avenue - San Francisco - California - USA 94112-1722

Phone: (415) 337-1100 E-Mail: service@astrosociety.org

Fax: (415) 337-5205 Web Site: www.astrosociety.org

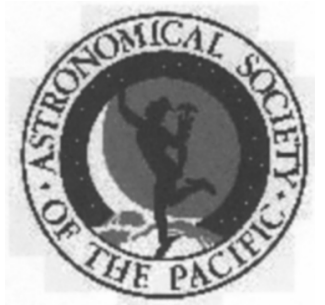

ASP-CS VOLUMES \& IAU PUBLICATIONS - EDITORIAL STAFF

Managing Editor: D. H. McNamara

Associate Managing Editor: J. W. Moody

Production Manager: Enid L. Livingston

PO Box 24463, Room 211 - KMB, Brigham Young University, Provo, Utah, 84602-4463

Phone: (801) 422-2111 Fax: (801) 422-0624 E-Mail: pasp@byu.edu

LaTeX-Computer Consultant: T. J. Mahoney (Spain)-tjm@ll.iac.es

A listing of all volumes published by the

Astronomical Society of the Pacific, is cited at the back of this volume 
INTERNATIONAL ASTRONOMICAL UNION

98bis, Bd Arago - F-75014 Paris - France

Tel: +33143258358 E-mail: iau@iap.fr

Fax: +33 143252616 Web Site: www.iau.org

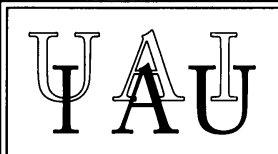

\title{
STAR FORMATION \\ AT HIGH ANGULAR RESOLUTION
}

\author{
Proceedings of the 221 th Symposium \\ of the International Astronomical Union \\ held during the IAU General Assembly XXV \\ Sydney, Australia \\ 22-25 July 2003
}

Edited by

MICHAEL G. BURTON

School of Physics, University of New South Wales

Sydney, NSW 2052, Australia

RAY JAYAWARDHANA

Department of Astronomy and Astrophysics, University of Toronto Toronto, Ontario M5S 3H8, Canada

and

TYLER L. BOURKE

Harvard-Smithsonian Center for Astrophysics, Submillimeter Array Project 645 N. A'ohoku Place, Hilo, Hawaii, 96720, USA 
C 2004 by International Astronomical Union All Rights Reserved

No part of the material protected by this copyright notice may be reproduced or utilized in any form or by any means - graphic, electronic, or mechanical including photocopying, taping, recording or by any information storage and retrieval system, without written permission from the IAU.

Library of Congress Cataloging in Publication Data

Main entry under title

LOC \#: $\quad 2003117078$

ISBN: $\quad 1-58381-161-3$

IAU Publications - First Edition

Published on behalf of the IAU by: Astronomical Society of the Pacific

Printed in United States of America by Sheridan Books, Ann Arbor, Michigan 


\section{Contents}

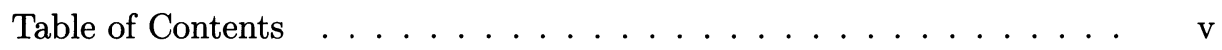

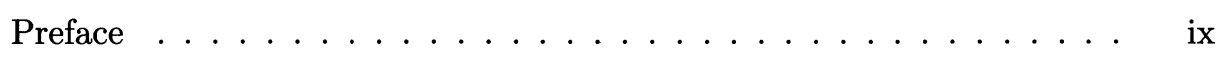

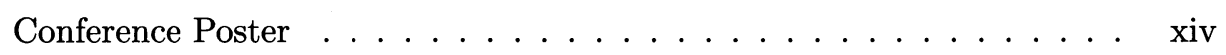

\section{Part 1. Star Formation}

Observations of Star Formation . . . . . . . . . . . . . . . 3

E. A. Lada and J. F. Alves

\section{Part 2. From Molecular Clouds to Protostellar Cores}

Techniques of mm/sub-mm Interferometry in Star Formation . . . . . . 19

$$
\text { A.I. Sargent }
$$

From Clouds to Protostellar Cores . . . . . . . . . . . . . . . 29 M. Saito

The Structure of Cold Molecular Cloud Cores . . . . . . . . . . . . 43

D. Ward-Thompson, D.J. Nutter, J.M. Kirk and P. André

The Turbulent Star Formation Model: Outline and Tests . . . . . . 51

E. Vázquez-Semadeni

Hot Molecular Cores and High Mass Star Formation . . . . . . . . . 59

F.F.S. van der Tak

Chemical Signatures of the Evolutionary State of Cores _ . . . . . 67

Y. Aikawa

Infall in Protostellar Envelopes . . . . . . . . . . . . . . . 75

N. Ohashi

Magnetic Fields in Molecular Clouds

T.L. Bourke and A.A. Goodman

Is the Magnetic Field Preserved During Core Formation? _ . . . . . . 97

B.C. Matthews, S.-P. Lai, R.M. Crutcher and C.D. Wilson 
Part 3. Extra-Galactic Star Formation

Extra-Galactic Star Formation Revealed . . . . . . . . . . . . . . . 107

E. Schinnerer

Molecular Gas and Star Formation in Nearby Galaxies . . . . . . . . . . 119

T. Wong and M.D. Thornley

Watching the Birth of Super Star Clusters . . . . . . . . . . . . 125

J.L. Turner and S.C. Beck

\section{Part 4. Massive Star Formation}

Masers: High Resolution Probes of Massive Star Formation . . . . . . . 133

S.P. Ellingsen

Theory of Formation of Massive Stars via Accretion . . . . . . . . . . 141

H.W. Yorke

The Most Detailed Picture Yet of an Embedded High Mass YSO . . . . 155

L.J. Greenhill, M.J. Reid, C.J. Chandler, P.J. Diamond and M. Elitzur

Formation and Early Evolution of Intermediate Mass Stars _. . . . . 161

L. Testi

Massive Star Formation . . . . . . . . . . . . . . . . . 169

G. Garay

High-Resolution Mid-Infrared Observations of High Mass Protostellar

Objects . . . . . . . . . . . . . . .

J.M. De Buizer

(Sub-)mm Interferometry in Massive Star Forming Regions . . . . . . . 191 H. Beuther

\section{Part 5. Low Mass Star Formation}

Testing the Paradigm of Low Mass Star Formation . . . . . . . . . . . 201

L. Hartmann

Observations of Binary Protostellar Systems . . . . . . . . . . . . 213

R. Launhardt

Infrared Studies of Protostellar Binaries . . . . . . . . . . . . . . . . 223

T. Greene

T Tauri Multiple Systems . . . . . . . . . . . . . . . . . . . . . . . 229

A. Brandeker

The Variation of the Initial Mass Function in Clusters . . . . . . . . . 237

K.L. Luhman

X-Rays From Class 0/I Protostars ～. . . . . . . . . . . . . . . 247

Y. Tsuboi

Theory of Young Clusters . . . . . . . . . . . . . . . . . . . 257

M.R. Bate and I.A. Bonnell 
The Structure and Kinematics of Protoclusters . . . . . . . . . . . . . 265 J. Di Francesco

\section{Part 6. First Results From New Facilities}

First Results from the ATCA at Millimetre Wavelengths . . . . . . . . . 275 V. Minier

Early Results from the SMA 283

Q. Zhang

VLTI-Early Results

C. Leinert

Studying Star Formation with the Keck Interferometer . . . . . . . . . . 301 R. Akeson

Adaptive Optics Imaging of Circumstellar Environments . . . . . . . . 307 D. Apai, I. Pascucci, H. Wang, W. Brandner, T. Henning, C. Grady and D. Potter

Subaru AO Coronagraphic and Direct Imaging of YSOs . . . . . . . . . 313 M. Tamura, M. Fukagawa, M. Hayashi and the SDPS/CIAO Teams

\section{Part 7. Jets and Outflows}

Adaptive Optics in Star Formation . . . . . . . . . . . . . . . . 323 W. Brandner

High Resolution Observations of Herbig-Haro Flows

B. Reipurth, S. Andrews, J. Bally and T.L. Beck

Outflow-Circumstellar Envelope Interactions in Protostars . . . . . . . . 345 H.G. Arce

Modeling Outflows from Young Stars . . . . . . . . . . . . . . . . . 351 H. Shang

\section{Part 8. Disks}

From Infall to Rotation around Young Stars:

The Origin of Protoplanetary Disks . . . . . . . . . . . . . 361 M.R. Hogerheijde.

High Resolution Optical and Infrared Images of Circumstellar Disks . . 373 D. Padgett and K. Stapelfeldt

High Angular Resolution Studies of Disks-the Millimetre . . . . . . . . 381 D.J. Wilner

Herbig Ae/Be Star Disks at High Angular Resolution . . . . . . . . . C.P. Dullemond, C. Dominik, R. van Boekel, R. Waters and M. van den Ancker

Early Disk Evolution . . . . . . . . . . . . . . . . . . . M. Wardle 
Models of Accretion Disks Around Young Stars . . . . . . . . . . . . . 403

P. D'Alessio, N. Calvet, L. Hartmann, J. Muzerolle and M. Sitko

Observations of Chemistry in Protoplanetary Disks . . . . . . . . . . . . 411 A. Dutrey

Probing AU-scale Structure using Spectro-astrometry . . . . . . . . . . 417

M. Takami, J. Bailey, A. Chrysostomou, M. Tamura and H. Terada

A Search for Disks around Massive Young Stellar Objects . . . . . . . . 425

A.G. Gibb, M.G. Hoare, L.G. Mundy and F. Wyrowski

\section{Part 9. The Influence of Planets During Star Formation}

Optical/IR Interferometry: Star Formation at sub-AU Scales . . . . . . 433 P. Tuthill

Detecting Planets within Disks

S. Wolf

ACS Coronographic Observations of Optically Thin Debris Disks

M. Clampin, J. Krist, D.R. Ardila, D.A. Golimowski, H.C. Ford and $G$. Illingworth

\section{Part 10. The Future of Star Formation at High Angular}

\section{Resolution}

Star Formation with Future Millimetre and Radio Interferometers . .

L.B.G. Knee

The Future of High Angular Resolution Star and Planet Formation Science in the Optical/Infrared . . . . . . . . . . . . . . .

L.A. Hillenbrand

Star Formation at High Angular Resolution: Summary and Outlook . .

H. Zinnecker

\section{Poster Papers, Program and Indexes}

List of Poster Presentations . . . . . . . . . . . . . . . . . . . . . . . . . 489

Conference Program . . . . . . . . . . . . . . . . . . . 499

Author Index ....................... . . . 505

Subject Index . . . . . . . . . . . . . . . . . . . . 507

Object Index ............................ 511 